\author{
Bill G. Felkey, MS, ${ }^{*}$ and Brent I. Fox, PharmD, PhD ${ }^{\dagger}$
}

\begin{abstract}
Those of you who have been in the health-system setting for several decades have seen many changes. Some of the changes originate within the care setting, whereas many others are brought on by external circumstances. Even those who have been in health systems for only 5 years can recall a recent change in the organization that greatly impacted pharmacy. More change is coming. In this installment, we explore critical technology-related changes of which you should be aware.
\end{abstract}

$\mathrm{H}$ ealth systems are increasingly finding themselves involved in something they describe as "straddling." They are straddling current reimbursement drivers and practices while concurrently getting ready for population health strategies to replace what has been the norm for decades. We have been saying for years that we cannot imagine a scenario where we will use less technology in any foreseeable future. When it comes to operating a health system focused on managing populations, we cannot imagine anything more important than the effective use of the following top 5 technologies.

\section{ELECTRONIC HEALTH RECORDS}

Of the 5 technologies highlighted in this column, electronic health records (EHRs) are, in our opinion, the hub for the other 4 and the most important to the success of health-system clinical operations within our current and future national health care system. To be clear, an electronic medical record can simply be an electronic version of a single provider's former, paper-based medical record. The EHR conforms to national interoperability standards. Although it documents the care of an individual, it can bridge across more than a single health care organization. An appropriately designed EHR can benefit cost, access, safety, quality, and effectiveness.

The essential components of the EHR include administrative processes that involve finances for the health care organization's operations. As we said, it is the hub for all communication and connectivity across the continuum of care. The best science is brought to bear through the use of decision support systems that promote best practices for each provider of care. Order entry management, patient support, and the ability to collect population data are necessary components. Documentation for quality improvement initiatives and reporting is also key. The system should support the management of current and historical information for each patient specifically and populations in general. Whenever possible, direct entry by each provider should be facilitated and simultaneous views by multidisciplinary care teams should be supported.

\section{HEALTH INFORMATION EXCHANGE}

Health information exchange (HIE) seeks to improve the speed, safety, quality, and cost of patient care by allowing all health care providers and patients to access important medical information electronically at every "handoff" of an individual patient within the continuum of care. Having the important information about a patient follow him or her throughout the care provision process will result in more appropriate and timely avoidance of readmission to hospitals, the reduction of medication errors, the decrease of duplicate testing, and the improvement of diagnoses.

HIE is currently taking place in 3 formats. Patient-mediated exchanges allow consumers to control how health information flows among providers (eg, the personal health record). Query-based exchanges allow providers to request patient information from other providers. Directed formats give providers the option to send and receive secure information in coordinated care programs. Directed

${ }^{*}$ Professor Emeritus, Auburn University, Auburn, Alabama; ${ }^{\dagger}$ Associate Professor, Department of Health Outcomes Research and Policy, Harrison School of Pharmacy, Auburn University, Auburn, Alabama 
formats are the most likely exchange method that will be used in population health management. Patient consent will be sought from all patient populations to facilitate exchanges in the most rapid access times possible.

\section{PATIENT PORTALS}

Patient portals differ from personal health records in several ways. Typically, a health system will create a branded portal that utilizes a browser-based or mobile app-based interface to transmit reports from episodes of patient care to include provider documentation and laboratory results. It will also open up a channel for patient reporting and inquiry. A personal health record will be employed by proactive patients to create a single location that can be accessed by any provider who is given access by the patient. Alternatively, personal health records can be promoted for use by patients in lieu of a health system creating its own portal.

We see patient portals as a key component to allow patient engagement and participatory health care to become a reality. Without self-care management behaviors taking place in patients' activities of daily living, it will be more difficult to achieve desired outcomes and contain costs. The components of involvement for patients will need to include shared decision making and respect for patient desires for treatment alternatives that match their individual needs. Provision will be have to be made for the interpretation of patient information that is transmitted from a personal health record or portal to ensure that vernacular errors are identified.

\section{TELEHEALTH}

Have you spent any time with FaceTime or Tango video phone calls? Have you seen the level of connectivity in the home of patients that is currently possible to facilitate remote patient monitoring? Are you aware of the level of wearable technology available to monitor health status and the lifestyle activity of patients? Did you know that the Internet of Things expects there to be 250 billion connected devices operating on our planet within the next 10 years?
Telehealth, telemedicine, telepharmacy, and telenursing will become key resources for population health management. Population health will require the rethinking of the level of primary care personnel we might expect to see. These communication channels will also be used for getting highly specialized providers involved in remote areas that would normally not support the practice of the specialist.

The technology for connecting homes to health care systems is not only doable, but is also affordable. Smart appliances allow for interaction that impacts many levels of health application. Smart phones contain sensors for vital sign measurement, and connected, affiliated partner devices allow for outcomes monitoring and can encourage increased levels of patient activity and other healthy behaviors. For example, fetal heart beat monitoring can be continuous throughout a pregnancy. There are hundreds of health technologies ready to be deployed.

\section{ADVANCED ANALYTICS}

Big data will be both the resource and the challenge for population health management. Risk stratification established by advanced analytics will allow the targeting and prioritization of resources within a population. Appropriate tracking and delegation within the continuum of care for patient action assignment and appropriate intervals for episodes of care will be generated by these analytics. Comparison data analysis on patient registries and implementation of patient engagement communication will be protocol driven within the population.

Best practices will be identified and promulgation of guidelines and flow sheets will occur as a byproduct of rendering patient care services. Other industries are managing lean processes and utilizing Six Sigma quality approaches to achieve desired results. Health care systems will need to apply similar processes to achieve the desired results across the population being served.

We would be happy to hear your comments and respond to your questions regarding this topic. You can contact Bill at felkebg@auburn.edu and Brent at foxbren@auburn.edu. 